\title{
MusX: Online Exploring and Visualizing Graph-Based Musical Adaptations
}

\author{
François Lévesque*, Marielle St-Germain ${ }^{\dagger}$, Dominique Piché*, Jean-François Gauvin ${ }^{\ddagger}$, \\ Michel Gagnon*, Thomas Hurtut* \\ ${ }^{*}$ Polytechnique Montréal, ${ }^{\dagger}$ EBSI, Université de Montréal, ${ }^{\ddagger}$ Bibliothèque et Archives nationales du Québec (BAnQ) \\ * francois.levesque, dominique.piche, michel.gagnon, thomas.hurtutepolymtl.ca \\ †marielle.st-germain@umontreal.ca, łjean-f.gauvin@banq.qc.ca
}

\begin{abstract}
This paper introduces MusX, a visualization-based system that helps to search and explore a large multivariate bipartite graph of artists and songs. An additional tree structure for the song nodes is also inherited from the musical adaptation relations. In tight collaboration with a public national library and archives institution, we propose a novel artist-centered interactive set of representations, focusing on several identified user tasks. This online system is targeted towards laypersons, willing to quickly navigate artists' body of songs and explore their relationships to other artists through their implication in song creation. In this paper, we present a detailed description of MusX along with design and technical considerations, and the demonstration scenarios we intend to present to the audience.
\end{abstract}

Keywords-bipartite graph; knowledge graph; exploration task; visualization.

\section{INTRODUCTION}

Open music databases such as MusicBrainz gather millions of songs and artists along with many metadata. These datasets quickly build up to large graphs with song nodes and artist nodes, which can be challenging to navigate, especially for laypersons [1]. This kind of dataset can also be curated by public library institutions which handle music collections and legal deposit. This paper presents a research work conducted in collaboration with such an institution, the Bibliothèque et Archives nationales du Québec (BAnQ), which is the national library and archives agency of the Quebec government. This institution has a dataset of around 24000 songs and 19000 artists.

In this dataset, a song gathers artists bearing three different possible roles (songwriter, composer, singer). Each role can be hold by multiple artists (e.g. if two songwriters coauthored a song). This dataset can thus be structured first into a bipartite graph, with artist nodes on a side, and song nodes on the other. Each edge in this graph encodes an artist role regarding a song, sometimes being multidimensional. For instance, Richard Coburn both wrote and composed the song Whispering, sung by Frank Sinatra. Besides, song lyrics can be adapted, either in another language, or simply into another lyrical version.This aspect naturally suggests a representation of a song as a tree structure where the root is the original song and each edge links a song to another song that is an adaptation The BAnQ dataset has a strong focus on this aspect since all songs have either a parent or a child node. The average number of songs per tree is 3.4 in the dataset, and the maximum depth of an adaptation tree is 4 . Following Munzner data abstraction [2], the BAnQ dataset is thus an unusual combination of a bipartite multivariate graph and an overlayed tree structure.

The design of the proposed demonstrated system conducted in collaboration with BAnQ, led to the proposition of an interactive visualization of this dataset, described in Section II. The proposed system targets several tasks. Overall, the intended users are the BAnQ website visitors, willing to explore the sometimes unexpected relations that may exist between artists and songs. BAnQ sees that exploration, or high-level consume task [2], as a possible stepping stone for a library borrowing of a physical compact disc. Specifically, given an artist, a user should be able to browse the distribution of his songs over time, and the songs from/to which they have been adapted. He should also be able to perform different kind of search actions on these bodies of songs, such as locate the artists with who their query collaborated the most and identify their roles, lookup, and identify artists' outliers, etc.

\section{SYSTEM OVERVIEW}

The client-server architecture of MusX is shown in Figure 1. It is composed of two main modules. (1) First a server uses a Resource Description Framework (RDF) model of the dataset. This model has been chosen for its easy interoperability with external linked open datasets such as MusicBrainz or DBPedia for data disambiguation or augmentation. The server side also proposes a SPARQL query endpoint which is a common querying interface for linked data model as RDF. (2) On the client side, a musical data exploration wall is based on three types of interactive visualizations depending on the user objective.

\section{A. Data model and query endpoint}

The RDF graph was built from a relational database, requiring various choices in regards to data structure. Authority records for artists and songs being the two main entities present in the original dataset, the RDF representation focuses on these two concepts. There are relationships between artists and songs (composition, authorship, performance), between songs (parent song, adapted song) and between authorities (members of a group, partnerships). The rest of the information is encoded with literal properties (dates, notes, etc.), or with external links (to the DBpedia pages for countries, 


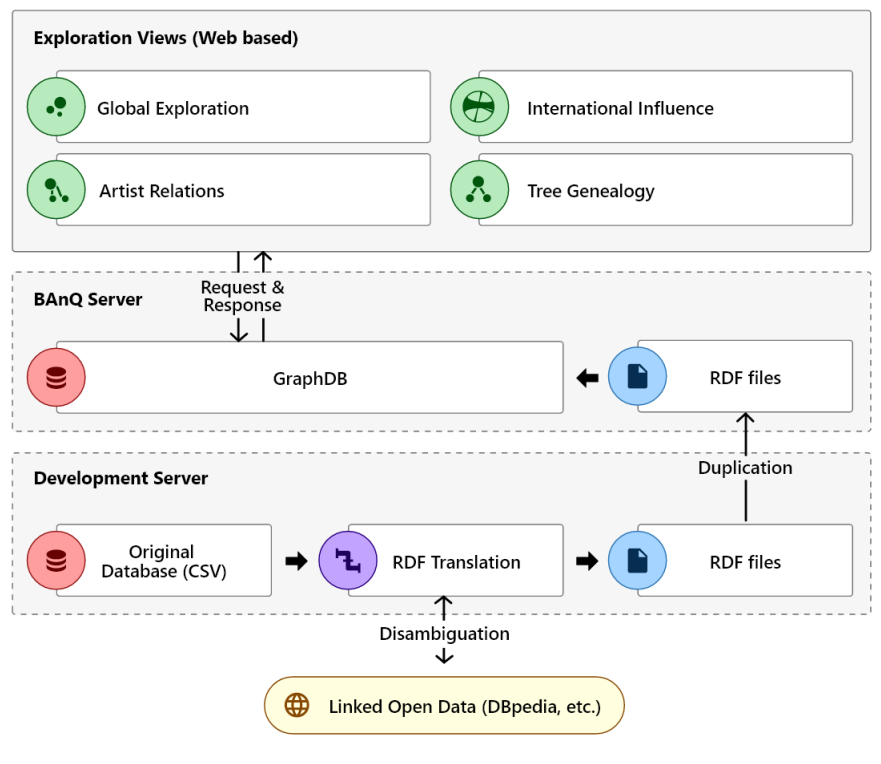

Fig. 1. System pipeline.

languages, genres and artists). Since the dataset was built in a library context, we chose to model the data according to the RDA Registry ${ }^{1}$. Resource Description and Access (RDA) is a standard used in cataloging specifying guidelines and rules on the description of bibliographic data. RDA Registry consists of vocabularies representing RDA elements, entities and terminologies in RDF. We also had to create new properties to represent a number of relationships. Finally, the RDF representation of the dataset is in a GraphDB triplestore ${ }^{2}$ and data is queried via a SPARQL endpoint.

\section{B. Exploration interface}

Overall, the intended users are the BAnQ website and library visitors. We have identified a set of 11 target questions that BAnQ users should be able to answer when exploring the dataset (see Table I). These questions were gathered from multiple sources: discussions, interviews and brainstorms with BAnQ collaborators, and a literature review on bipartite graphbased visualization systems [3, 4].

Questions Q1 to Q3 seek to explore the whole dataset from an artist perspective. For instance it helps discover which artists are the most prolific in terms of adaptations, either because they adapted a lot of songs, or because they inspired many artists to adapt theirs. Q3 seeks to characterize a possible correlation between these last two aspects. Questions Q4 seeks to explore the adaptation flows by aggregating adaptations along the main country from which the involved artists are operating. Thus, it endeavors to characterize the relations between countries reflected through the number of adaptations going from one to the other. Questions Q5 and Q6 seek to understand the whole, and sometimes unexpected, genealogy of a song in terms of adaptations, and their lyrics languages. Questions Q7 to Q10 are transverse and artist-centered. They

\footnotetext{
${ }^{1}$ https://www.rdaregistry.info/

${ }^{2}$ https://www.ontotext.com/products/graphdb/
}

TABLE I

QUESTIONS FOR THE ANALYSIS OF SONG ADAPTATIONS DATA.

1 Which artists are the most popular/inspirational in terms of their songs being adapted by others?

2 Which artists have adapted the most songs compared to their whole body of songs?

3 Do artists who often adapt songs tend to be inspirational, that is create songs that are often adapted?

4 What songs of an artist have been adapted to/from a specific country?

5 What is the genealogy of a song in terms of all its adaptations?

6 In how many languages a song has been adapted?

7 When do we find highest number of adaptations over time for a given artist?

8 Which songs of an artist have been adapted over time?

9 Which songs an artist has adapted over time?

10 What are the relations of an artist with others through the songs in which he has been involved?

11 What are the artists involved in the songs adapted from/to a given artist songs?

seek to characterize how a specific artist's body of songs is structured in terms of adaptations. As such, they sustain the characterization of when, and from/towards who an artist's relations have been oriented along his career. In order to attain these targeted questions, the exploration interface is based on four main exploration views, each targeting specific user questions and scenarios.

Artists global view. This view is shown in Figure 2. It can be used as an entry point into the system since it does not need any user input. It covers questions Q1 to Q3. The design is based on a bubble-chart where each disk represents an artist, encoding his number of songs as the disk's surface. The X-axis encodes the percentage of his songs that have been adapted by other artists. The Y-axis encodes the percentage of his songs that are adaptations. The user can navigate this view with panning, zooming and filtering interactions, search for an artist with a search box, and click on a disk to open the single artist exploration wall (Figure 3). As such, this view rely on the Shneiderman's mantra: "overview first, zoom and filter, then details-on-demand" [5].

Relationship exploration view. This view, shown in Figure 3(a), targets questions Q7 to Q11. Given a chosen artist $A$, this view enable the exploration of the subset of all the songs in which this artist is directly involved (thereafter called level 0 songs), their potential direct parent songs in terms of musical adaptation (level -1 songs), and their potential direct children songs (level +1 songs). In other words, level 0 songs represent the body of songs of $A$, level -1 songs are the songs at the origin of an adaptation in which $A$ is involved, and level +1 songs are adaptations from songs in which $A$ is involved. As such it characterizes the level of bidirectional inspiration of an artist. In the main top panel, three timelines gather the songs from each of these level, allowing an exploration of this inspiration aspect, over the temporal dimension. In the bottom panel, for each level, we represent all the other artists involved, along with their roles, in tabular panel lists also containing small barcharts of their relative number of implications. Hovering interactions give 


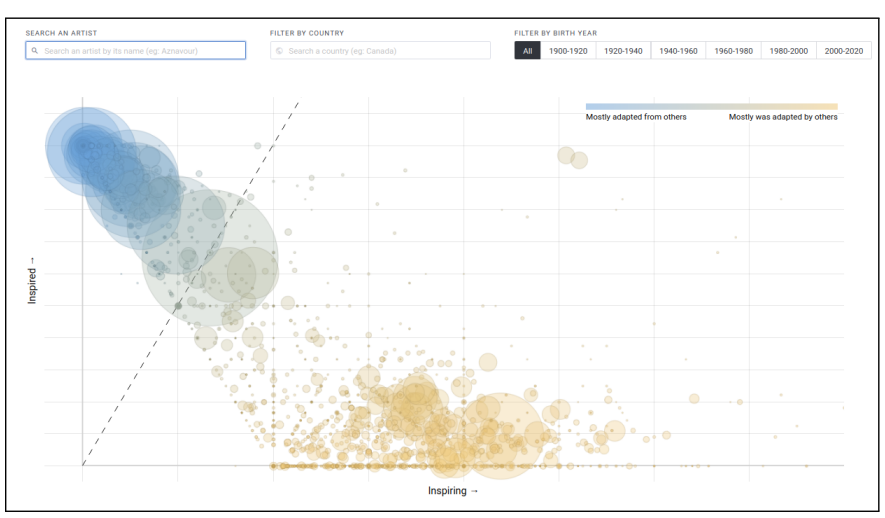

Fig. 2. Artists global view.

linked information between the panels such as the set of songs of a specific artist who has collaborated with $A$ in the level 0 tabular list. Every artist visible in the view is clickable, leading to its own relationship exploration view, in order to foster user exploration.

The node link design was chosen for its expressive topological representation. The attribute-driven grouping allows to quickly reflect the relationship nature between songs [6]. Then, adding edges effectively creates connected timelines. All edges are drawn with a low opacity and a small bezier curve in order to reduce the visual clutter when the graph is dense. With such a layout, it becomes difficult to distinguish individual trees, but the accumulation of edges provides rich visual cues regarding the trend and distribution of the relationships.

Country pairwise flow views. This view, shown in Figure 3(b), targets questions $\mathrm{Q} 4$. For a given artist $A$, it shows the same songs as in the relationship exploration view, yet reorganised along the directional adaptation flows between the countries associated with the artists implied in the song. These flows are visualized with two chord diagrams, a first one for the level -1 to level 0 adaptations (characterizing from which countries are the songs that $A$ adapted), and a second one for the level 0 . to level +1 adaptations (characterizing to which countries $A$ 's songs were adapted). A chord diagram presents a radial design where a chord width encodes the number of songs adapted from a country to another. The user can filter the songs according to the time period, or its artist role, and click a chord to see the list of associated songs.

Song genealogy view. This view, shown in Figure 3(c), targets questions Q5 and Q6. If a user clicks on a song, either in the relationship exploration view or the country pairwise flow view, he can visualize the full tree genealogy of a song along with the language associated to each version lyrics.

\section{RELATED SYSTEMS AND NOVELTY}

Before starting the work on MusX, we identified a number of exploratory and semantic search systems developed in the wake of the proliferation of studies on Semantic Web technologies since the early 2000s: Aemoo $^{3}$ [7], Discovery $\mathrm{Hub}^{4}$ [8] and Linked $\mathrm{Jazz}^{5}$ [9]. These served as inspiration and sources of discussion for our own system. It should be noted, however, that the number of exploratory and semantic search systems is relatively limited and systems described in papers are often no longer updated or accessible. In these examples, we identified different functionalities and specifications meeting the definition we acknowledged of an exploratory and semantic search system : the presentation of results, data provenance and query suggestions. Aemoo and Linked Jazz both allow users to explore the results in a graph while Discovery Hub presents the results of queries in the more traditional form of a list. A particularly interesting feature of Linked Jazz is the fact that the user can access and navigate in the whole dataset as a graph, allowing an exploration of jazz musicians and their relationships. On the flip side, the size of the dataset, and therefore the graph, makes navigation trickier and overwhelming for some users. This observation had an impact on our project due to the important number of relationships (there are 1106021 total statements in the MusX triplestore). Also, since library and archives users are used to seeing information displayed in lists and in bibliographic records, it was important in this project to propose different possibilities for data visualization that would support the user in his learn and investigate activities. Another important point is the fact that the development of the Semantic Web technologies and Linked Open Data principles led to a different way of thinking about the management and dissemination of information. This is particularly interesting in the context of exploratory search systems because the creation of semantic equivalences between concepts as well as the potential for data enrichment through the linking of data coming from heterogeneous and external sources can support the development of solutions appropriate in situations where the user does not search for a particular document or piece of information and where interoperability between systems is an important benefit. In the case of Aemoo, Discovery Hub and Linked Jazz, DBpedia plays a central role as a data source and as a tool for semantic reconciliation. MusX uses DBpedia for disambiguation of certain entities. Finally, since we are in an exploratory search context, it was important to offer query suggestions that would be a relevant starting point for the users. In Discovery Hub, a tool generates random results, proposing an alternative to the traditional entry of a keyword in a search box. Aemoo, for its part, proposes different search examples (e.g. Silvio Berlusconi, Mickey Mouse, Galileo Galilei). MusX builds on these examples and offers different entry points to the data : through search examples, and the global artist view.

\section{DEMONSTRATION SCEnARIOS}

An online implementation is available at http://musx.witify. io. The tool has been developed with the $d 3 . j s$ and vue.js

\footnotetext{
${ }^{3}$ http://wit.istc.cnr.it/aemoo/index.html

${ }^{4}$ http://discoveryhub.co/

${ }^{5}$ https://linkedjazz.org/
} 


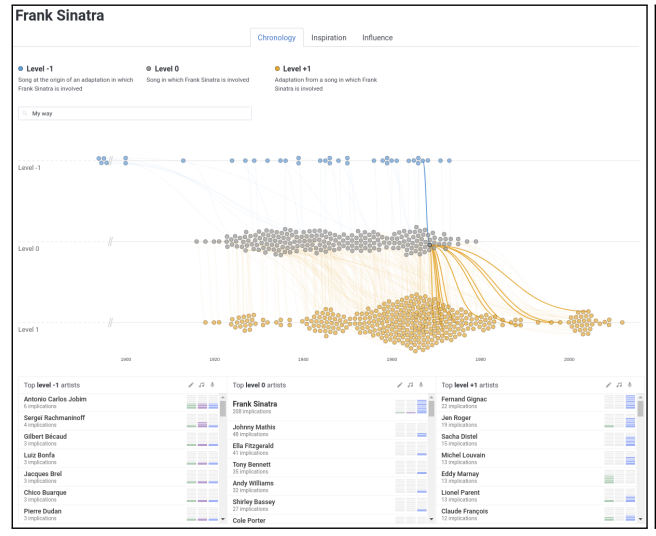

(a) Relationships exploration view

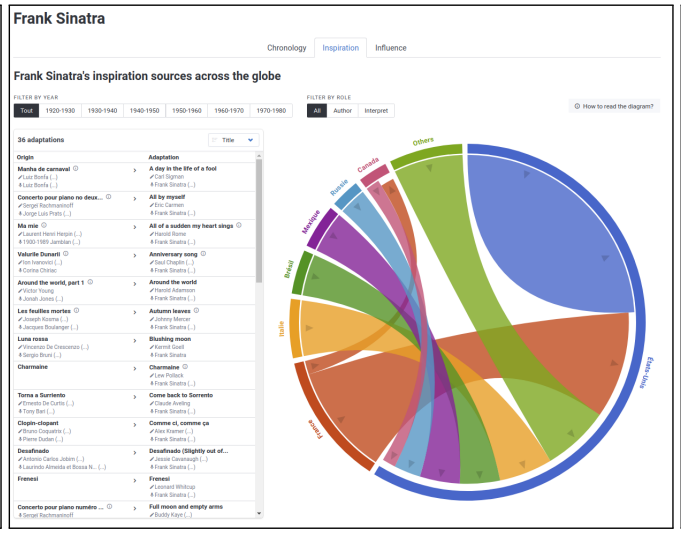

(b) Country pairwise flow view

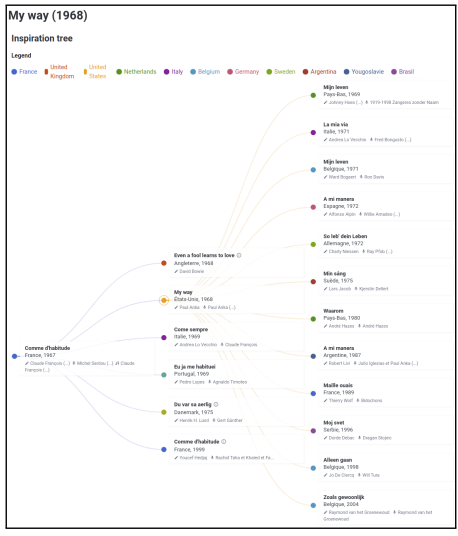

(c) Song genealogy view.

Fig. 3. Single artist exploration wall.

libraries, entirely in javascript. The key objectives of the intended demonstration are to enable the audience to interactively experience the following scenarios, related to the identified questions and tasks detailed in Section II.

Scenario 1: Overall exploration of whole set of artists We will first present a use-case scenario targeting questions Q1 to Q3 from Table I. Using the artists global view, the user will be able to identify which artists are the most popular and prolific in terms of adaptations, compared to the total number of songs they were implied in. We will demonstrate how a user can visualize the whole distribution of artists, estimate the correlation between the two adaptation directions, navigate and zoom in specific patterns of the distribution, identify outlier artists, filter artists based on the period of time and country, and get details on demand by clicking and eventually moving on to a different view.

Scenario 2: Dive into an artist body of songs and relations Once an artist has been chosen, we will then show how a user can visualize the sub-part of the bipartite graph to which he pertains, using the relationship exploration view (Figure 3(a)). This scenario will focus on questions Q7 to Q11 from Table I. We will demonstrate how a user can explore and discover the relations of the chosen artist to others through adapted songs, over time, depending on the artists' role. We will also show how a user can explore an artist's body of songs and his own roles held along his career. We observed in preliminary studies that in such scenario, unplanned fortunate discoveries, also called serendipity findings, can frequently occur. Non-expert users are often not fully aware of the adaptation relations that pertain to an artist they know, especially when they involve international influences.

Scenario 3: A given artist international influence This scenario targets questions Q4 to Q6. A user having a specific artist in mind (possibly chosen from the first scenario), we will show how he can visualize his international influence and inspiration in terms of adaptation flows between countries (Figure 3(b)), eventually focusing on some specific years, country pairs, and languages through the use of the song genealogy (Figure 3(c)).

\section{ACKNOWLEDGMENT}

This work was supported by a grant from the Secrétariat du Conseil du Trésor du Québec in collaboration with BAnQ, and a NSERC grant (RGPIN-2015-06025).

\section{REFERENCES}

[1] R. Pienta, J. Abello, M. Kahng, and D. H. Chau, "Scalable graph exploration and visualization: Sensemaking challenges and opportunities," in Proc. Int. Conf. on Big Data and Smart Computing (BIGCOMP), 2015, pp. 271-278.

[2] T. Munzner, Visualization analysis and design. AK Peters Visualization Series, CRC Press, 2014.

[3] I. Herman, G. Melançon, and M. S. Marshall, "Graph visualization and navigation in information visualization: A survey," IEEE Transactions on Visualization and Computer Graphics, pp. 24-43, 2000.

[4] T. Von Landesberger, A. Kuijper, T. Schreck, J. Kohlhammer, J. J. van Wijk, J.-D. Fekete, and D. W. Fellner, "Visual analysis of large graphs: state-of-the-art and future research challenges," Computer Graphics Forum, vol. 30, no. 6, pp. 1719-1749, 2011.

[5] B. Shneiderman, "The eyes have it: A task by data type taxonomy for information visualizations," in Proc. IEEE Symposium on Visual Languages, 1996, pp. 336-343.

[6] C. Nobre, M. Streit, M. Meyer, and A. Lex, "The state of the art in visualizing multivariate networks," Computer Graphics Forum (Proc. EuroVis), pp. 807-832, 2019.

[7] A. G. Nuzzolese, V. Presutti, A. Gangemi, P. Musetti, and P. Ciancarini, "Aemoo: exploring knowledge on the web," in Proc ACM Web Science Conference, 2013, pp. 272-275.

[8] N. Marie, F. Gandon, M. Ribière, and R. Florentin, "Discovery hub : on-the-fly linked data exploratory search," in Proc. ACM Int. Conf. on Semantic Systems, 2013.

[9] M. Miller, J. Walloch, and M. C. Pattuelli, "Visualizing linked jazz: A web-based tool for social network analysis and exploration," Proc. American Society for Information Science and Technology, vol. 49, no. 1, pp. 1-3, 2012. 